\title{
Is Graphene Oxide a Chemoattractant?
}

\author{
Chengdong Zhang ${ }^{*}+, \S$, Yaqi Wang ${ }^{\ddagger}$, , and Huiru Zhao ${ }^{\ddagger}$ \\ $\dagger$ School of Environment, Beijing Normal University, Xin Jie Kou Wai ST 19, Beijing 100875, \\ China \\ $\$$ College of Environmental Science and Engineering, Nankai University, Tong Yan Rd. 38, \\ Tianjin 300350, China \\ *E-mail: zhangchengdong@bnu.edu.cn \\ $\S$ These authors contributed equally to this work.
}




\section{Experimental Procedures}

\subsection{Materials.}

Graphene oxide (GO), GO-1, GO-2 and GO-3 were purchased from different companies in China (Plannano, Suzhan, Chengdu Organic Chemicals, and XFNANO), respectively. The solvent used in this study was phosphate buffer saline (PBS, ionic strength $161.7 \mathrm{mM}, \mathrm{pH} 7.0$ ) (Thermo Fisher Scientific, US). Potassium ethylenediaminetetraacetate (EDTA-3K) was purchased from Yuanye, China. Diaminonaphthalene (DAN) was obtained from Aladdin, China. A 4, 5-dihydroxypentane-2,3-dione (DPD) solution (AI-2 precursor, $4.47 \mathrm{mM}$ ) was purchased from Omm Scientific (US). The capillaries were purchased from Drummond Scientific (US). The $\mu$-Slide Chemotaxis ${ }^{3 \mathrm{D}}$ was obtained from IBIDI (Germany). The syringe ( $1 \mathrm{~mL}$ ) was acquired from Becton Dickinson (US). Sterilized deionized water was used, and all chemical reagents were analytical grade or better.

\subsection{Characterization of GO samples.}

The surface elemental composition was measured by X-ray photoelectron spectroscopy (XPS) (Thermo Scientific ESCALAB 250Xi, Thermo, US). The particle size distribution (20 $\mathrm{mg} / \mathrm{L}$ in PBS) and surface charge were determined by dynamic light scattering (DLS) using a ZetaPALS (Brookhaven Instruments, Holtsville, US). The extent of disorder was determined by Raman spectroscopy (RM2000, London, UK). The graphene sheet was visualized by transmission electron microscopy (TEM) (JEM-2800, Japan) at a $100 \mathrm{kV}$ accelerating voltage.

\subsection{Bacterial strain, $\Delta t s r$ mutant construction and culture conditions.}

Escherichia coli K12 MG 1655 (E. Coli) has been widely used as a model microbe for studying chemotaxis..$^{1-3}$ In this study, E. coli was purchased from the Agricultural Culture Collection of China and routinely cultured in Luria-Bertani broth (LB containing $0.5 \% \mathrm{NaCl}$, 
$0.5 \%$ yeast extract, and $1 \%$ peptone; $\mathrm{pH} 7.0)$ with continuous shaking $(160 \mathrm{rpm})$ at $37{ }^{\circ} \mathrm{C}$. Bacteria in the exponential phase of growth (approximately $5 \mathrm{~h}$ growth) were used in all subsequent experiments.

The mutant $\Delta t s r$ was constructed by Abace-biology (China). Briefly, $\Delta t s r$ was generated by the CRISPR/Cas9 genomic editing system according to the literature. ${ }^{4,5}$ The primer sequences are listed as below. Successful construction was confirmed by genomic PCR and sequencing as shown below.

\begin{tabular}{ll}
\hline \multicolumn{1}{c}{ Primer } & \multicolumn{1}{c}{ Sequence (5'-3') } \\
\hline sgRNA-F & CCTTTTACAACTGACATCAGGTTTTAGAGCTAGAAATAGC \\
sgRNA-R & CTGATGTCAGTTGTAAAAGGACTAGTATTATACCTAGGAC \\
TsrU-F & TTACGGCGAGAGGAGATAAACAG \\
TsrU-R & CGGGCATTTTCATGGCGAGGTTTCTCTTTCCTGTGGACCG \\
TsrD-F & TCCACAGGAAAGAGAAACCTCGCCATGAAAATGCCCGATAAG \\
TsrD-R & ATCGGCTGGTATATGCTCTATCG \\
Yz1-F & CGACCTACACCGAACTGAGA \\
Yz1-R & CTGATGTCAGTTGTAAAAGG \\
Yz2-F & ATGTTAAAACGTATCAAAATTGT \\
Yz2-R & TTAAAATGTTTCCCAGTTCTCCT \\
\hline
\end{tabular}

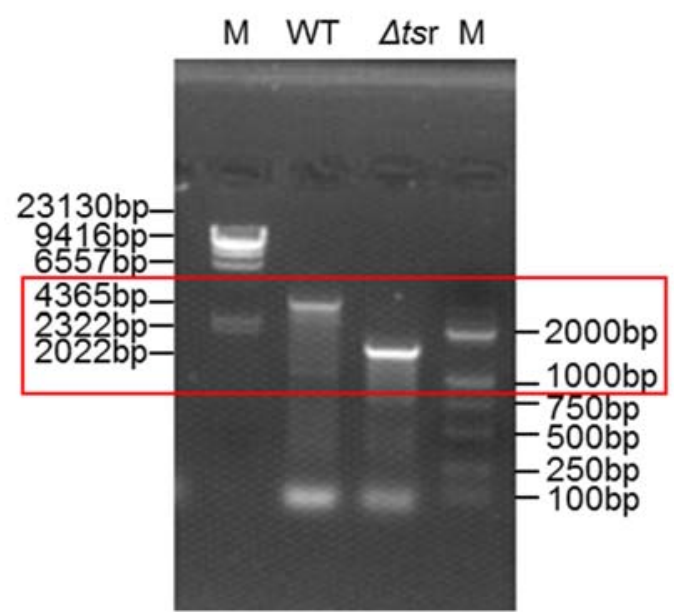


TsrU-F: TTACGGCGAGAGGAGATAAACAG

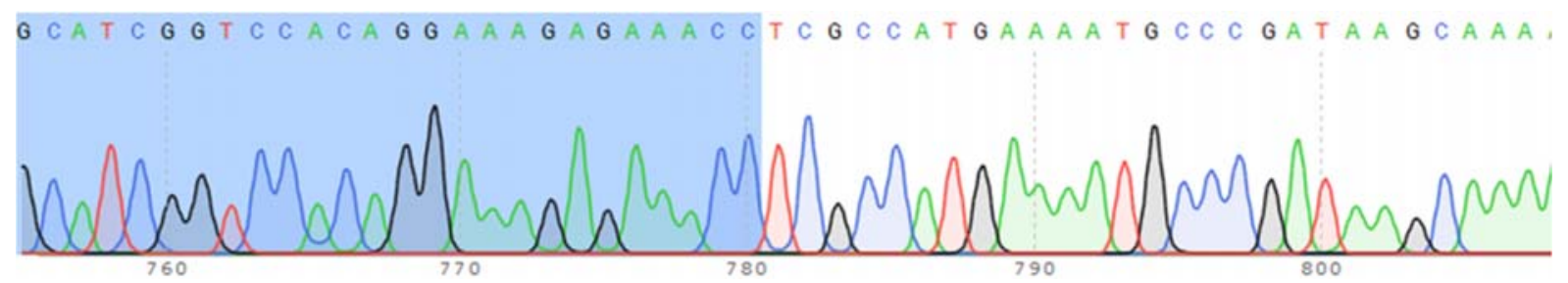

TsrD-R: ATCGGCTGGTATATGCTCTATCG

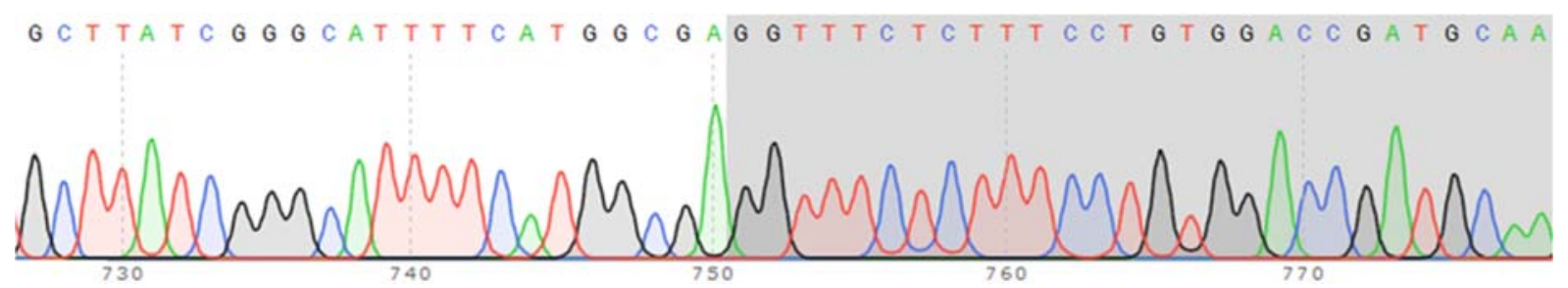

\subsection{Method.}

\subsubsection{Capillary assay.}

The capillary tube was heated at the melting point and bent to form a U-shape. The bacterial chamber was composed of a U-shaped tube, a cover glass and a slide. Another capillary with one sealed end was filled with $0.5 \mu \mathrm{L}$ of $20 \mathrm{mg} / \mathrm{L}$ nanomaterial suspension prepared in chemotactic medium (PBS with $0.01 \mathrm{mM}$ EDTA-3K). ${ }^{6}$ The bacterial suspension in chemotactic medium (with a density of $10^{7} \mathrm{CFU} / \mathrm{mL}$ ) was added to the bacterial chamber. After 15 minutes, the capillary was withdrawn, and the outer surface was washed several times with water to remove bacteria attached to the outside of the capillary. Then, the liquid inside capillary was expelled onto a petri dish, and after culturing overnight, the number of colonies was counted. To film the movement, the capillary device was placed on a confocal laser scanning microscope (LSM880 with Airyscan, Zeiss, Germany), and the movements of bacteria near the capillary orifice were recorded continuously during the first 6 minutes. ${ }^{7}$ The image was obtained at the interval of $600 \mathrm{~ms}$, and 600 cycles were performed. The movie was played at a speed of 60 frames per second. ${ }^{8}$ The tracing of motile bacterial and statistical analysis of moving direction 
and speed was processed with NIS-Elements AR Analysis. The capillary assay was performed in at least triplicate.

\subsubsection{Microfluidic chip assay.}

The bacterial suspensions were stained with the green fluorescent nucleic acid stain SYTO 9 (Thermo Fisher Scientific, US) ${ }^{9}$ according the manufacturer's instructions. The labeled bacteria were resuspended in chemotactic medium at a density of $10^{6} \mathrm{CFU} / \mathrm{mL}$. The fluorescent bacterial suspension $(10 \mu \mathrm{L})$ was added into the center stream. Then, $60 \mu \mathrm{L}$ of chemotactic medium was added to both chambers from the injection orifice, while $15 \mu \mathrm{L}$ of $40 \mathrm{mg} / \mathrm{L}$ nanomaterial in chemotactic medium was added only to the left chamber. To accelerate diffusion, $15 \mu \mathrm{L}$ of solution was withdrawn by pipet from the extraction port, which was located opposite of the injection orifice. The process was repeated one more time. ${ }^{10}$ The distribution of bacterial suspension was visualized by confocal laser scanning microscopy. The assay by microfluid chip was repeated in at least triplicate.

\subsubsection{Syringe assay.}

The syringe tube was filled with $200 \mu \mathrm{L}$ of $20 \mathrm{mg} / \mathrm{L}$ nanomaterial (prepared in chemotactic medium), and the syringe needle was immersed into a bacterial suspension (with a density of $10^{7}$ $\mathrm{CFU} / \mathrm{mL}$ in chemotactic medium), which was contained in an Eppendorf tube. The plunger was fixed tightly to prevent sliding. The setup was maintained upright for 15 minutes. ${ }^{11}$ Then, the needle was removed, and the suspension inside the syringe tube was expelled. After appropriate dilution, the bacterial suspension was inoculated on a petri dish. The number of colonies was counted after culturing overnight. When needed, different types/concentrations of GO were used inside the syringe, or $\Delta t s r$ was used instead of wild-type $E$. coli. If needed, $20 \mathrm{mg} / \mathrm{L}$ or $5 \mathrm{~g} / \mathrm{L}$ of glucose was added to the bacterial suspension. To test the effect of temperature, the bacterial 
suspension was immersed in iced water. The experiments were performed in at least triplicate, and the results are expressed as the mean \pm standard deviation.

\subsubsection{Transcriptome sequencing.}

The mid-exponential phase bacteria were exposed to 5 or $20 \mathrm{mg} / \mathrm{L} \mathrm{GO}$ in $0.9 \% \mathrm{NaCl}$ solution for 30 minutes. Then, the cells were harvested by centrifugation at $8,000 \times \mathrm{g}$ for 10 minutes at $4{ }^{\circ} \mathrm{C}$, flash frozen in liquid nitrogen, and stored at $-80{ }^{\circ} \mathrm{C}$ until RNA was extracted. $E$. coli alone was used as a biotic control. Total RNA was extracted and purified using the RNA simple Total RNA Kit (Tiangen Biotech, China) according to the protocol provided by the manufacturer. RNA purity was checked using the NanoPhotometer ${ }^{\circledR}$ Spectrophotometer (IMPLEN, US) and RNA integrity was assessed using the RNA Nano 6000 Assay Kit of the Agilent Bioanalyzer 2100 system (Agilent Technologies, US). The cDNA libraries were constructed using the NEBNext Ultra ${ }^{\mathrm{TM}}$ RNA Library Prep Kit (New England Biolabs, US) following the manufacturer's instructions with a total of $3 \mu \mathrm{g}$ RNA per sample as input material. After the mRNA was purified and fragmented, clustering and sequencing were conducted by Novogene Co. (Beijing, China) using IlluminaTruSeq PE Cluster Kit v3-cBot-HS (Illumina, US) with two technical repeats.

Raw reads in FASTQ format were processed by in-house perl scripts. Raw reads in FASTQ format were processed to obtain clean reads by trimming the adaptor sequences out of the reads using Trimmomatic software v0.33 and filtering the reads as follows: (a) reads with adaptor sequences, (b) low quality reads (where the number of bases with $Q \leq 20$ accounts for more than $50 \%$ of the entire read length), and (c) reads with high percentages of unknown bases (where unknown bases account for more than $10 \%$ of the entire read length). Consequently, the Q20, Q30, and GC contents of the clean data were calculated. Quality control information is 
provided in Table S1 and S2. Index of the reference genome was built using Bowtie v2.2.3 and paired-end clean reads were aligned to the reference genome using TopHat v2.0.12. HTSeq v0.6.1 was used to count the read numbers mapped to each gene. Then, fragments per kilobase of transcript per million mapped reads (FPKM) of each gene was calculated based on the length of the gene and reads count mapped to this gene. And differentially expressed genes (DEGs) were analyzed with DESeq R package (1.18.0). The DEGs between two samples were identified by considering both the fold change $\left(\log _{2} \mathrm{FC}>1\right)$ and the $p$-value $(<0.005)$. The Gene Ontology (GO) term enrichment of the DEGs was conducted using the GOseq R package. The statistical enrichment of DEGs in Kyoto Encyclopedia of Genes and Genomes (KEGG) pathways was tested by using KOBAS software.

\subsubsection{Quantitative real-time polymerase chain reaction (qRT-PCR).}

The regulation of key genes involved in chemotaxis, PTS and flagella assembly was quantified via qRT-PCR analysis. The exponential phase cells were exposed to $20 \mathrm{mg} / \mathrm{L}$ of GO or glucose in $0.9 \% \mathrm{NaCl}$ solution for 30 minutes. Total cellular RNA was extracted using TRIzol reagent (Invitrogen, US), and the FastKing RT Kit (With gDNase) (Tiangen, China) was used for reverse transcription. PCR was then performed in triplicate using SuperReal PreMix Plus (SYBR Green) (Tiangen, China) according to the manufacturer's instructions on a QuantStudio TM6 Real-Time System (Life Technologies, Singapore) with a $20 \mu \mathrm{L}$ reaction system. The thermal cycle started at $95{ }^{\circ} \mathrm{C}$ for 15 minutes and then ran for 40 cycles of $95{ }^{\circ} \mathrm{C}(10 \mathrm{~s})$ and $60{ }^{\circ} \mathrm{C}(30 \mathrm{~s})$, followed by a melting curve analysis post run. The mRNA expression levels were calculated and normalized against the housekeeping gene 16S rRNA using the $-\Delta \Delta \mathrm{C}(\mathrm{T})$ method. ${ }^{12}$ The primers used for qRT-PCR were designed by Sangon Biotech (China) and are listed in Table S3. The 
experiments were performed in at least triplicate, and the results are expressed as the mean \pm standard deviation.

\subsubsection{Autoinducer 2 (AI-2) assay.}

The exponential phase cells were collected and resuspended in $10 \mathrm{~mL}$ PBS to reach a density of $10^{10}-10^{11} \mathrm{CFU} / \mathrm{mL}$. Then, the cells were exposed to $20 \mathrm{mg} / \mathrm{L}$ GO for $30 \mathrm{~min}$. After centrifugation at $5000 \mathrm{rpm}$ for 10 minutes, the supernatant was mixed with DAN solution (10 mg dissolved in $50 \mathrm{~mL} 0.1 \mathrm{M} \mathrm{HCl}$ ) at a 1:1 ratio and vortexed for 2 minutes. The mixture was incubated in a $90{ }^{\circ} \mathrm{C}$ water bath for 40 minutes. After cooling, $50 \mu \mathrm{L}$ samples were analyzed by High Performance Liquid Chromatography (Waters e2695, US) equipped with a multi $\lambda$ fluorescence detector (Waters 2475, US). Separation was achieved on a Unitary C18 reversephase column $(250 \mathrm{~mm} \times 4.6 \mathrm{~mm}, 5 \mu \mathrm{m})$ set at $30{ }^{\circ} \mathrm{C}$. The mobile phase was composed of acetonitrile (solvent $\mathrm{A}$ ) and $0.1 \%$ formic acid solution (solvent $\mathrm{B}$ ), and the flow rate was 0.8 $\mathrm{mL} /$ minute. The gradient elution profile was as follows: $\mathrm{t}=0$ minutes, $30 \%$ solvent $\mathrm{A}, 70 \%$ solvent $\mathrm{B} ; \mathrm{t}=4$ minutes, $30 \%$ solvent $\mathrm{A}, 70 \%$ solvent $\mathrm{B} ; \mathrm{t}=12$ minutes, $65 \%$ solvent $\mathrm{A}, 35 \%$ solvent $\mathrm{B} ; \mathrm{t}=20$ minutes, $65 \%$ solvent $\mathrm{A}, 35 \%$ solvent $\mathrm{B} ; \mathrm{t}=24$ minutes, $30 \%$ solvent $\mathrm{A}, 70 \%$ solvent $\mathrm{B}$; and $\mathrm{t}=27$ minutes, $30 \%$ solvent $\mathrm{A}, 70 \%$ solvent $\mathrm{B}$. The excitation and emission wavelengths of the fluorescence detector were $271 \mathrm{~nm}$ and $503 \mathrm{~nm} .{ }^{13}$ The cells that were not exposed to GO were used as biotic controls, and the AI-2 standard (590 ng/mL in PBS) was used to verify the retention time and to quantify the relative concentration. The experiments were performed in at least triplicate, and the concentration was expressed as the mean \pm standard deviation.

\subsection{Statistical analysis.}


All experiments were performed in at least triplicate. The data were expressed as the means \pm standard deviation, and significant differences were determined by $t$-test or one-way ANOVA. A $p$-value less than 0.05 indicated a significant difference. 


\section{Supplementary Figures and Tables}
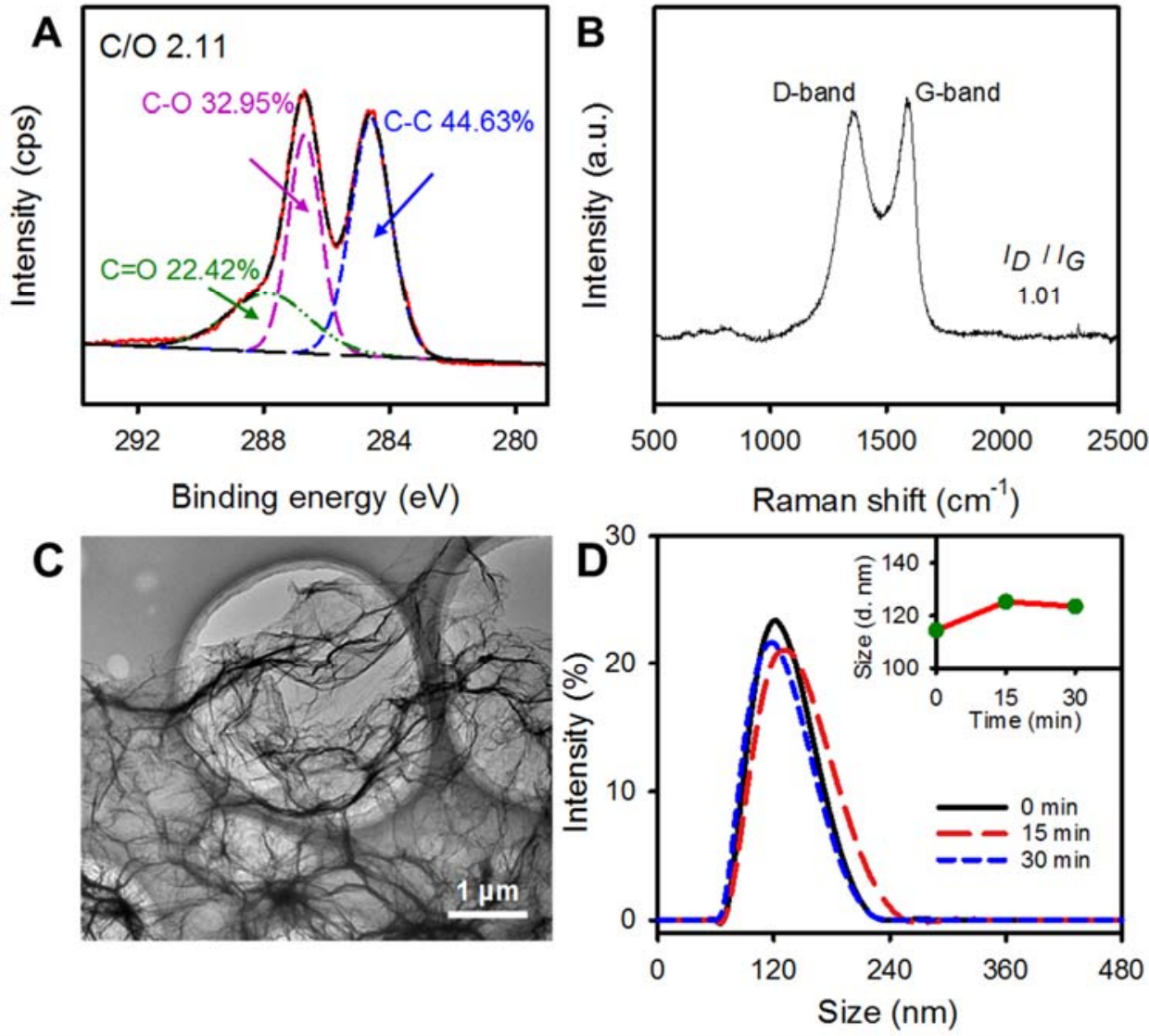

Figure S1 (A). XPS spectra of C1s for GO. The peaks with binding energies of 284.6, 286.6, and $287.9 \mathrm{eV}$ were assigned to the carbon atoms in the aromatic rings $(\mathrm{C}-\mathrm{C} / \mathrm{C}=\mathrm{C})$, the epoxy/hydroxyl $(\mathrm{C}-\mathrm{O}-\mathrm{C} / \mathrm{C}-\mathrm{OH})$ groups, and the carbonyl $(\mathrm{C}=\mathrm{O}) /$ carboxyl $(\mathrm{O}-\mathrm{C}=\mathrm{O})$ groups, respectively. The relative content of each functional group is indicated. (B) Raman spectra of $\mathrm{GO}$; the $I_{D} / I_{G}$ ratio is annotated. (C) TEM image of GO $(20 \mathrm{mg} / \mathrm{L})$ dispersed in PBS. (D) Size distribution of GO $(20 \mathrm{mg} / \mathrm{L})$ over time in PBS analyzed by DLS. The insert shows the changes in hydrodynamic diameter over time and the error bars were smaller than the symbols in this case. The experiments were carried out in at least triplicate. The results were expressed as the mean \pm standard deviation. 

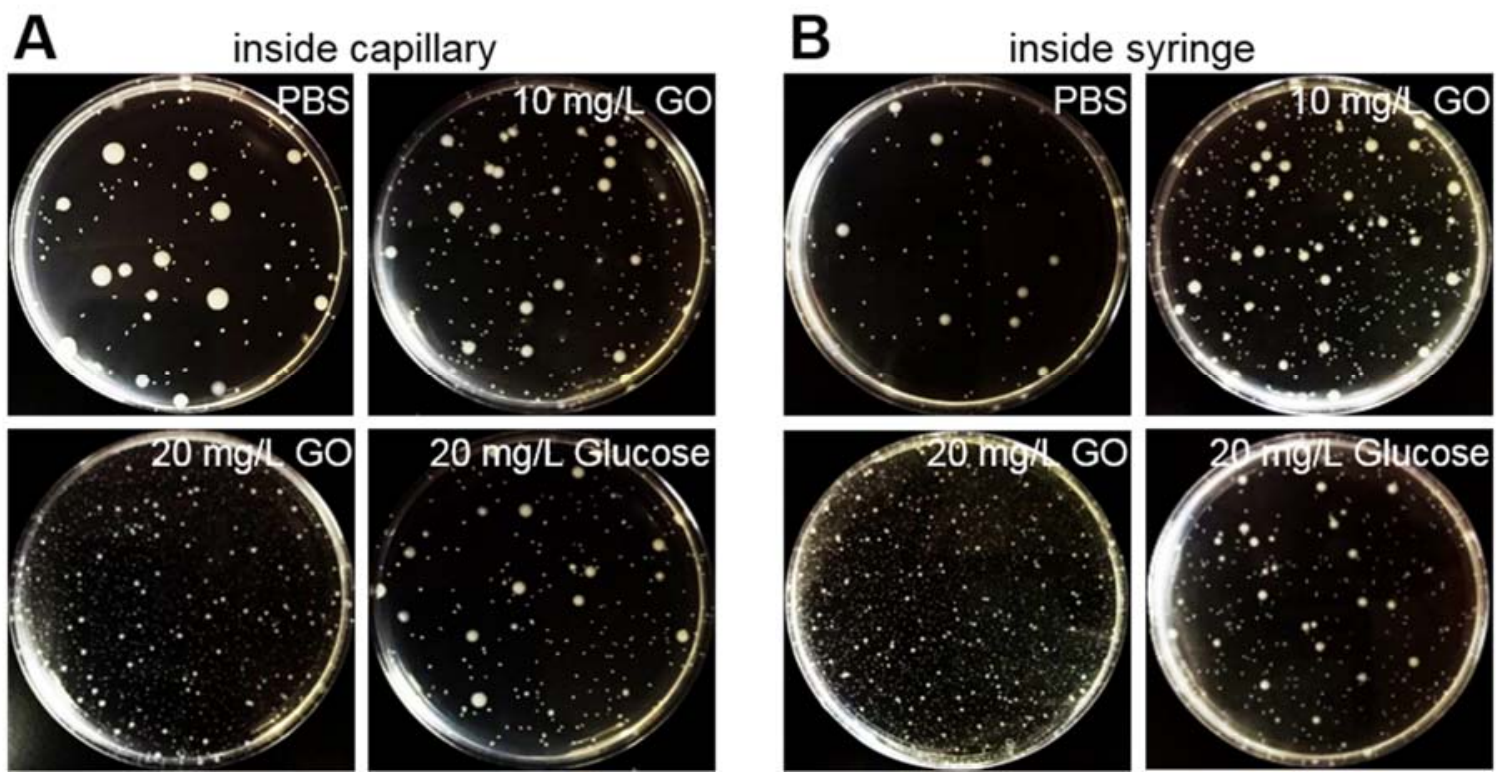

Figure S2. Visual comparisons of $E$. coli colonies under different treatments based on the (A) capillary assay and (B) syringe assay. Different concentrations of GO (10 or $20 \mathrm{mg} / \mathrm{L})$ or 20 $\mathrm{mg} / \mathrm{L}$ glucose were used as attractant after incubation for 15 minutes. After proper dilution in PBS, the bacteria inside capillary/syringe were enumerated on the plate and incubated for $12 \mathrm{~h}$. Bacteria also moved into capillaries or syringes in the absence of chemoattractants due to diffusion. The photographs were taken with a smart phone. 
The Most Enriched Gene Ootology Terms (upregulated)

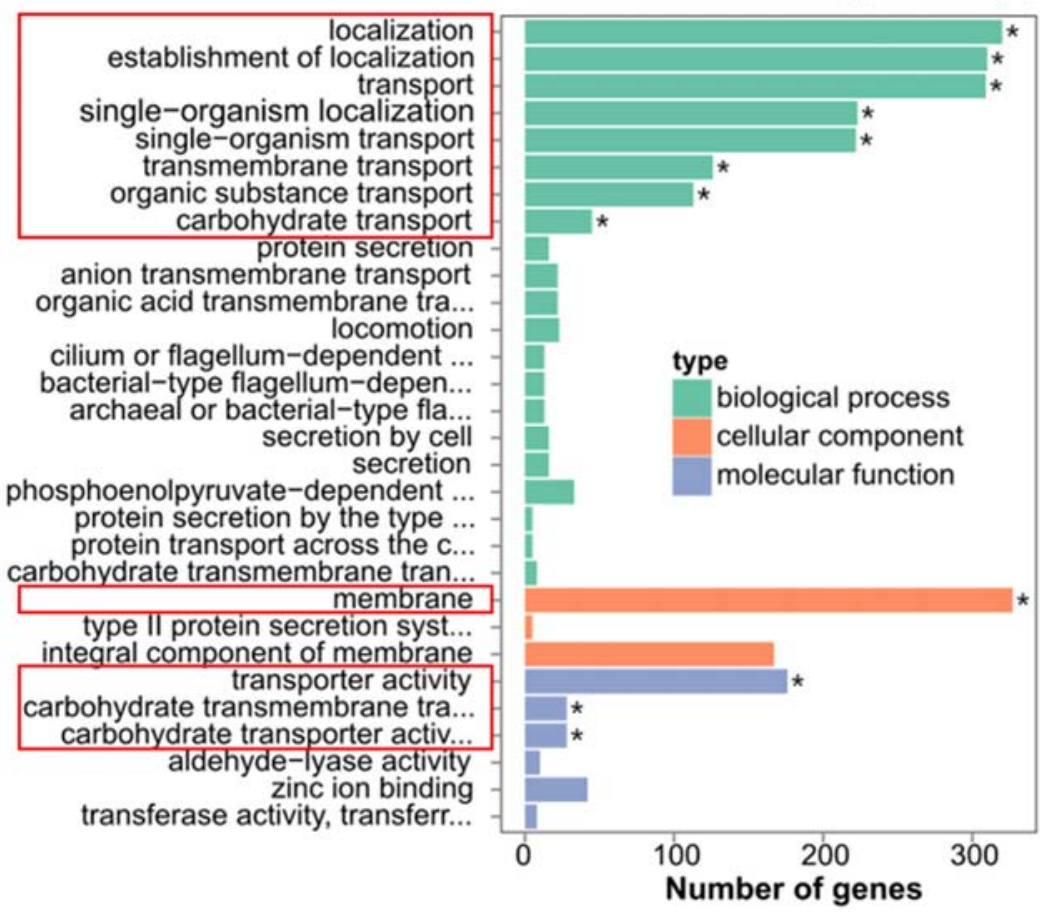

Figure S3. The most enriched Gene Ontology terms annotated for significantly upregulated genes. E. coli cells were exposed to $20 \mathrm{mg} / \mathrm{L} \mathrm{GO}$ in $0.9 \% \mathrm{NaCl}$ solution for 30 minutes and cells alone were used as biotic control. The red rectangular box highlights that the most affected bacterial activity was membrane-related transport processes. 

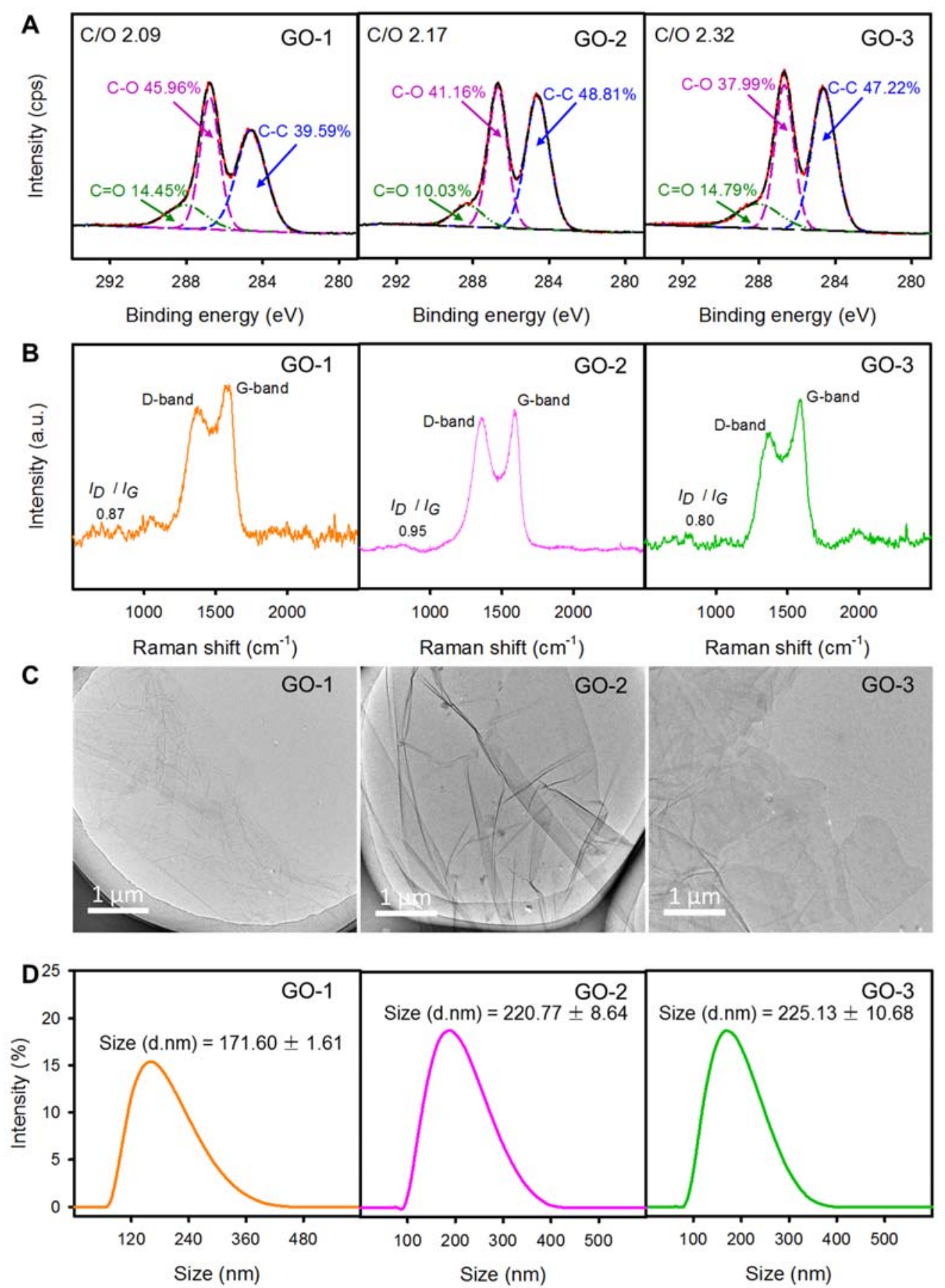

Figure S4. Characterization of different GOs by (A) XPS, (B) Raman spectroscopy, (C) TEM, and (D) DLS analysis (20 mg/L in PBS after 15 minutes of incubation). The experiments were carried out in at least triplicate. The results are expressed as the mean \pm standard deviation. 
Table S1. Sequencing and assembly statistics for the transcriptomic data.

\begin{tabular}{|c|c|c|c|c|}
\hline Sample & Raw reads & Clean reads & Total mapped ${ }^{[\mathrm{a}]}$ & $\begin{array}{l}\text { Uniquely } \\
\text { mapped }^{[b]}\end{array}$ \\
\hline Control & 8759596 & 8759596 & $\begin{array}{l}8378883 \\
(95.65 \%)\end{array}$ & $\begin{array}{l}7970165 \\
(90.99 \%)\end{array}$ \\
\hline $5 \mathrm{mg} / \mathrm{L} \mathrm{GO}$ & 15804534 & 15061626 & $\begin{array}{l}14237063 \\
(94.53 \%)\end{array}$ & $\begin{array}{l}13276598 \\
(6.38 \%)\end{array}$ \\
\hline $20 \mathrm{mg} / \mathrm{L} \mathrm{GO}$ & 7876576 & 7876576 & $\begin{array}{l}7445131 \\
(94.52 \%)\end{array}$ & $\begin{array}{l}7042087 \\
(88.15 \%)\end{array}$ \\
\hline
\end{tabular}

[a] Total mapped is the number of clean reads that mapped to the reference genome.

${ }^{[b]}$ Uniquely mapped is the number of clean reads that mapped to the reference genome at only one site. 
Table S2. The distribution of genes in differentially expressed levels.

\begin{tabular}{llll}
\hline FPKM $^{[\mathrm{a}]}$ interval & Control & $5 \mathrm{mg} / \mathrm{L} \mathrm{GO}$ & $20 \mathrm{mg} / \mathrm{L} \mathrm{GO}$ \\
\hline $0 \sim 1$ & $936(18.74 \%)$ & $796(15.94 \%)$ & $650(13.01 \%)$ \\
$1 \sim 3$ & $399(7.99 \%)$ & $322(6.45 \%)$ & $48(0.96 \%)$ \\
$3 \sim 15$ & $946(18.94 \%)$ & $930(18.62 \%)$ & $316(6.33 \%)$ \\
$15 \sim 60$ & $1102(22.06 \%)$ & $1110(22.22 \%)$ & $1681(33.65 \%)$
\end{tabular}

${ }^{[a]}$ FPKM is the expected number of fragments per kilobase of transcript sequence per million base pairs sequenced. 
Table S3. The primers used in qRT-PCR analysis and their target classification ${ }^{[\mathrm{a}]}$.

\begin{tabular}{|c|c|c|c|}
\hline Primer names & Forward primer $\left(5^{\prime}-3^{\prime}\right)$ & Reverse primer $\left(5^{\prime}-3^{\prime}\right)$ & $\begin{array}{l}\text { Gene } \\
\text { classification }\end{array}$ \\
\hline 16S rRNA & TCCTACGGGAGGCAGCAG & GGACTACCAGGGTATCTAATCCTGTT & \\
\hline tsr & ACCATTCGCCAGCAGCAATCC & CACTCTCCATCAGCTCAGCAACG & \multirow{10}{*}{ Chemotaxis } \\
\hline $\operatorname{tar}$ & TGAAGTGCGTAATCTTGCCAGTCG & TTCATCCGATGCCGATGCAATCTC & \\
\hline tap & GAGTCAGTCAATCGTGTCCAGCAG & CTGTTGTTCTTCCGACGCAGAGG & \\
\hline $\operatorname{trg}$ & TCACCGCCGTCATCAACAGTATTG & GCGACTTGCGAGTGTCCGTAC & \\
\hline $\operatorname{mot} \mathrm{A}$ & CACCGCCTATCGCCGTTGAG & TCCTCGGTTGTCGTCTGTTGTTG & \\
\hline cheA & TGCATTGAAGTGACCGACGATGG & TGACCTGCTCTGCCGTGGAG & \\
\hline cheB & GCCTTGCAGCACATAAGCCATTG & ACGTGACGAATTGCCTCAGTTCC & \\
\hline cheZ & TTGCCGATCCGATTGACCTTGC & CTGGCGTTCGATCTCCTGAATGAC & \\
\hline cheY & AATGTTGAGGAAGCGGAAGATGGC & TAACACTGGCAATGCCGACATCG & \\
\hline cheR & CTAATCAGCACAGCGGTGAGTGG & AATGTGTCAGCCAGCGTCATCG & \\
\hline flhB & CTGCTGGTGGTGCTTGGTGTC & GCTCGCTGCATCTGACGGATC & \multirow{5}{*}{$\begin{array}{l}\text { Flagellar } \\
\text { assembly }\end{array}$} \\
\hline fliN & GATGACAACAACGGCGCAATGG & ATATCCTGCAACGTTCCGCTGAC & \\
\hline fliM & GACGAACCGACAGCCAGTGTTAG & ACGACGCAGCAGGTTGAACAG & \\
\hline fliG & GAATGGCTTGCTCGACGGTCAG & CGGCGGTAATAACGGCTTCTTCC & \\
\hline fliE & GCGATACAGGGGATTGAAGGG & AACTAATGGTCGGTTGCGGC & \\
\hline
\end{tabular}




\begin{tabular}{lll|}
\hline flgH & GGTAACGCTCGTGCCGATGTC & CTGGTCAACCGTCACCGTCAAC \\
\hline flgF & TCCAACCTTGCGTGTGATGTCG & GCTGGCAATCATGTCGCTCATTG \\
\hline fliK & CGCCGTCAACCGTGTTACCG & TCAGGCTGTGCTGTTGTGAGTTG \\
\hline malX & CTCGGCGTCACCATCGGTAATAC & CCACTGGCACCATGTACCACTTG \\
\hline ascF & CGCTGATCGGCACCATGTCAC & AGCACCGTCACCAATCACATTCAG \\
\hline cmtA & CCGGTTGGCTGCCTAACGAAC & TACCGCCGACCAGATGACCAC \\
\hline agaV & GGTCAGGTCGGCGTTCAATGG & CGGATCTTCGGCAACCTCATCG \\
\hline gatA & AGGCGATTCATGCTAAGTCGTCAG & ACCGCCACGTCGTTATCATCATC \\
\hline fruA & GGCGATTATGGCGGCAGGTATG & GGCAGCACACGCATCGGATC \\
\hline ulaC & GCAGGCTGAAGCAGAGACATGG & ACCGAACTGTTCAACGCCATCC \\
\hline [a] $\mathrm{Tat}$ & & \\
\hline
\end{tabular}

${ }^{[a]}$ Target gene description was found by BLAST in the National Center for Biotechnology Information (NCBI) databases. 


\section{References}

(1). Typas, A.; Sourjik, V., Bacterial protein networks: properties and functions. Nat. Rev. Microbiol. 2015, 13, 9, 559-572.

(2). Sourjik, V.; Wingreen, N. S., Responding to chemical gradients: bacterial chemotaxis. Curr. Opin. Cell Biol. 2012, 24, 2, 262-268.

(3). Minamino, T.; Imada, K., The bacterial flagellar motor and its structural diversity. Trends Microbiol. 2015, 23, 5, 267-274.

(4). Jiang, Y.; Chen, B.; Duan, C. L.; Sun, B. B.; Yang, J. J.; Yang, S., Multigene Editing in the Escherichia coli Genome via the CRISPR-Cas9 System. Appl. Environ. Microbiol. 2015, 81, 7, 2506-2514.

(5). Joung, J.; Konermann, S.; Gootenberg, J. S.; Abudayyeh, O. O.; Platt, R. J.; Brigham, M. D.; Sanjana, N. E.; Zhang, F., Genome-scale CRISPR-Cas9 knockout and transcriptional activation screening. Nat. Protoc. 2017, 12, 4, 828-863.

(6). Adler, J., A method for measuring chemotaxis and use of the method to determine optimum conditions for chemotaxis by Escherichia coli. J. Gen. Microbiol. 1973, 74, 1, 77-91.

(7). Jain, R.; Faith, N. G.; Milkowski, A.; Nelson, K.; Busche, D.; Lynn, D. M.; Czuprynski, C. J.; Abbott, N. L., Using Chemoattractants to Lure Bacteria to Contact-Killing Surfaces. Angew.

Chem., Int. Ed. 2016, 55, 19, 5698-5702.

(8). Meijering, E.; Dzyubachyk, O.; Smal, I., METHODS FOR CELL AND PARTICLE

TRACKING. In Imaging and Spectroscopic Analysis of Living Cells: Optical and Spectroscopic Techniques, Conn, P. M., Ed. Elsevier Academic Press Inc: San Diego, 2012; Vol. 504, pp 183200.

(9). Musken, M.; Di Fiore, S.; Romling, U.; Haussler, S., A 96-well-plate-based optical method for the quantitative and qualitative evaluation of Pseudomonas aeruginosa biofilm formation and its application to susceptibility testing. Nat. Protoc. 2010, 5, 8, 1460-1469.

(10). Hotz, M.; Nelson, W. J., Pumilio-dependent localization of mRNAs at the cell front coordinates multiple pathways required for chemotaxis. Nat. Commun. 2017, 8.

(11). Shu, L.; Zhang, B.; Queller, D. C.; Strassmann, J. E., Burkholderia bacteria use chemotaxis to find social amoeba Dictyostelium discoideum hosts. ISME J. 2018, 12, 8, 1977-1993.

(12). Li, M. Z.; He, Y. N.; Sun, J.; Li, J.; Bai, J. H.; Zhang, C. D., Chronic Exposure to an Environmentally Relevant Triclosan Concentration Induces Persistent Triclosan Resistance but Reversible Antibiotic Tolerance in Escherichia coli. Environ. Sci. Technol. 2019, 53, 6, 32773286.

(13). Song, X.-N.; Qiu, H.-B.; Xiao, X.; Cheng, Y.-Y.; Li, W.-W.; Sheng, G.-P.; Li, X.-Y.; Yu, H.-Q., Determination of autoinducer-2 in biological samples by high-performance liquid chromatography with fluorescence detection using pre-column derivatization. J. Chromatogr. A 2014, 1361, 162-168. 